\title{
INFLUÊNCIA DA CONFIGURAÇÃO DA SEN NO COMPORTAMENTO FLUIDODINÂMICO EM MOLDE DE BEAM BLANK
}

\author{
Weslei Viana Gabriel ' \\ Johne Jesus Mol Peixoto ' \\ Gesiane Letícia Alves ${ }^{2}$ \\ Carlos Antonio da Silva ${ }^{3}$ \\ Itavahn Alves da Silva ${ }^{3}$ \\ Varadarajan Seshadri ${ }^{4}$
}

\section{Resumo}

Utilizando técnicas de modelagem física e matemática comparou-se o fluxo de fluido no interior do molde de beam blank alimentado por apenas uma válvula submersa, com 3 e 4 portas na saída respectivamente. Na válvula com 4 portas, observou-se que para um diâmetro da porta de saída inferior de $10 \mathrm{~mm}$ não houve alterações significativas no comportamento do fluxo, comparado à válvula com apenas três portas laterais. Ao aumentar o diâmetro para $16 \mathrm{~mm}$, houve alteração significativa do perfil de velocidade, reduzindo a assimetria de fluxo, fato que pode contribuir para a redução no gradiente térmico ao longo do molde, diminuindo a possibilidade de ocorrência de defeitos no material lingotado. Com o aumento da dimensão da porta inferior observou-se também redução na velocidade de impacto do jato principal e consequentemente reduziu-se a velocidade do fluxo ascendente, o que evita refusão da pele e entranhamento de escória. Palavras-chave: Lingotamento contínuo; Válvula submersa; Beam Blank; Fluxo de fluido.

\section{INFLUENCE OF SEN CONFIGURATION IN BEAM BLANK CAST FLUIDODYNAMIC BEHAVIOR}

\begin{abstract}
Physical and mathematical simulations was used to characterize the fluid flow inside a beam blank mold fed with one submerged entry nozzles containing three and four ports respectively. Comparing both systems was observed that when this fourth port had diameter equal $10 \mathrm{~mm}$ no significantly changes could be seen in relation to the SEN without this port. Increasing the diameter to $16 \mathrm{~mm}$ change the velocity profile significantly, reducing the asymmetry of the flow decreasing the thermal gradient in the mold and consequently the possibility of defects occurrence on the product. Was observed too the reduction of impingement velocity with the higher diameter of the base port. This reduce the velocity of the ascendant flow, avoiding remelting of the skin and slag entrainment.
\end{abstract}

Keywords: Continuous casting; SEN; Beam blank; Fluid flow.

\section{INTRODUÇÃO}

Existem diversas configurações de sistemas para alimentação do molde de beam blank utilizando válvulas submersas, podemos agrupá-los em dois grupos: os sistemas compostos por duas válvulas posicionadas nos flanges e os com apenas uma colocada em um dos flanges. Peixoto et al. [I] mostraram que a utilização de apenas uma válvula tubular propicia uma grande assimetria de fluxo e alta penetração do jato e que a utilização de duas válvulas tubulares reduz esta assimetria, porém não soluciona a alta penetração do jato. Esta alta penetração prejudica a fusão do pó fluxante

'Programa de Pós-graduação, Rede Temática em Engenharia de Materias - REDEMAT, Universidade Federal de Ouro Preto - UFOP, Ouro Preto, MG, Brasil.E-mail: wesleiviana@yahoo.com.br

${ }^{2}$ Escola de Minas, Universidade Federal de Ouro Preto - UFOP, Ouro Preto, MG, Brasil.

${ }^{3}$ Departamento de Engenharia Metalúrgica e de Materiais, Escola de Minas, Universidade Federal de Ouro Preto - UFOP, Ouro Preto, MG, Brasil.

${ }^{4}$ Escola de Engenharia, Universidade Federal de Minas Gerais, Belo Horizonte, MG, Brasil.

*Versão revisada de trabalho apresentado na ABM Week 20I7, de 2 a 6 de outubro de 20I7, São Paulo, SP, Brasil.

2176-1523 (C) 2017 Associação Brasileira de Metalurgia, Materiais e Mineração. Publicado pela ABM. Este é um artigo de acesso aberto distribuído sob os termos da licença Creative Commons CC BY-NC-ND (Attribution-NonCommercial-NoDerivs) - https:// creativecommons.org/licenses/by-nc-nd/4.0\%. 
(Zhang et al. [2]) e dificulta a limpidez do aço (Onishi et al. [3] e Gabriel et al. [4]).

Para solucionar problemas oriundos da alta penetração do jato em sistemas com duas válvulas, Yang et al. [5] e Chen et al. [6] dentre outros propuseram um modelo de válvulas submersas constituídos por três portas laterais na saída. Este modelo apresentou baixa penetração do jato e maior turbulência no menisco. Xuo e Zhu [7] relatam que esta configuração permite desenvolver um fluxo de fluido e de calor mais uniforme gerando uma casca solidificada de espessura mais homogênea, além de intensificar a oscilação superficial.

Porém, para evitar dificuldades operacionais para controle de vazão e posicionamento da SEN, aliada ao desejo de redução nos custos, as instituições optam pela utilização de apenas uma válvula. Por isto, é interessante avaliar as características de fluxo desenvolvidos por diferentes modelos, de modo a desenvolver um sistema de alimentação mais simplificado e que propicie melhor produtividade da máquina de lingotamento. Este trabalho compara o padrão de fluxo desenvolvido em um sistema de alimentação com apenas uma válvula submersa, contendo na saída três e quatro portas respectivamente.

\section{MATERIAIS E MÉTODOS}

Foram realizadas simulações físicas e matemáticas do fluxo de fluido no interior de um molde com formato de beam blank. As velocidades de lingotamento empregadas foram 0,78, 0,98 e I, $2 \mathrm{~m} / \mathrm{min}$. A profundidade de imersão da válvula foi de $250 \mathrm{~mm}$. A caracterização do fluxo foi realizada através da análise do perfil de velocidade, dispersão de traçador e medição da oscilação superficial. $\mathrm{Na}$ modelagem física utilizou-se as técnicas PIV (Particle Image Velocimetry), injeção de traçador e análise superficial com sensor ultrassônico.

Para modelamento matemático foi utilizado o software Ansys CFX 17.I. Considerou-se a água como um fluido newtoniano, temperatura constante e igual a $25^{\circ} \mathrm{C}$. O software resolve as equações da continuidade, de Navier-Stokes e da viscosidade efetiva, além das equações auxiliares do modelo de turbulência $k-\varepsilon$ para determinação de $k$ (energia cinética de turbulência) e $\varepsilon$ (taxa de dissipação de energia cinética de turbulência). Como critério de convergência, adotou-se a condição em que todos os resíduos relativos às equações de fluxo, estivessem inferiores a $1 * 10^{-6}$.

Os elementos de malha aplicados são tetraédricos na maior parcela do corpo e hexaédrico na região da interface água - ar, conforme apresentado por Gabriel et al. [8].

A válvula submersa aplicada possui 3 portas laterais (uma porta com $47 \mathrm{~mm}$ de diâmetro e duas portas com $16 \mathrm{~mm}$ ). Avaliou-se também a influência da inserção de uma quarta porta na base. A Figura I apresenta os modelos de válvula submersa estudados, as regiões avaliadas no molde para caracterização do fluxo bem como as condições de contorno aplicadas no modelamento matemático. No plano AA avaliou-se o perfil de velocidade através das técnicas PIV e CFD. Na linha I mediu-se a velocidade horizontal do jato, comparando o resultado obtido por ambas as técnicas. Nos planos BB e CC avaliou-se a velocidade máxima obtida por modelamento matemático. Para análise da dispersão de traçador no fluido via CFD, realizaram-se simulações computacionais em regime transiente, timestep de 0,005 s e tempo total de 30 s. O traçador foi adicionado

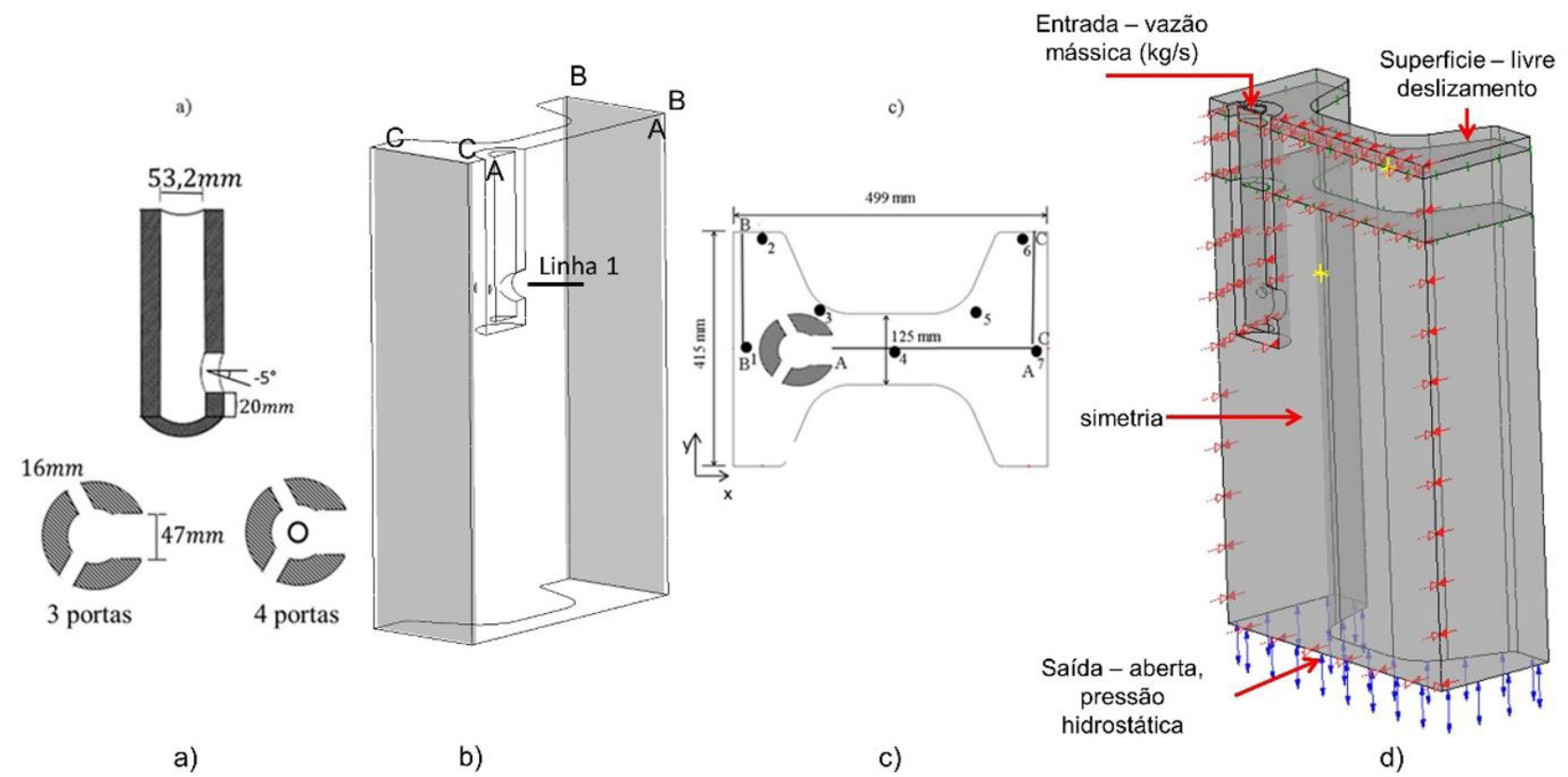

Figura I. a) Válvulas submersas, b) Molde, destacando as regiões analisadas, c) seção transversal do molde de beam blank e pontos de análise de oscilação superficial, d) condições de contorno aplicados no modelamento matemático. 
pela técnica de Additional Variable, permitindo a inserção de um componente não reativo que acompanha o fluxo sem interferir no mesmo. Resultados da simulação permanente foram utilizados como condição inicial.

\section{RESULTADOS E DISCUSSÃO}

Para validar os resultados obtidos através do modelamento matemático, comparou-se o perfil de velocidade com o obtido pela técnica PIV. A Figura 2 apresenta valores de velocidade ao longo da linha I para as técnicas PIV e CFD, comparando a influência da inserção de um quarto furo na válvula submersa com diâmetro de $10 \mathrm{~mm}$.

A porta inferior, com diâmetro de $10 \mathrm{~mm}$, não exerceu efeito significativo no perfil de velocidade quando comparado ao sistema composto por apenas 3 portas. A técnica PIV determina o perfil de velocidade a partir do acompanhamento da trajetória de partículas dispersas no fluido ao longo do plano (AA) iluminado pelo laser pulsante. A curvatura dos filetes provoca reflexos do feixe de laser e distorções nas imagens. Além disso, a saída de partículas na direção normal ao plano analisado resulta em acentuadas variações do valor instantâneo de velocidade. Estes fatores contribuem para as flutuações observados no perfil médio de velocidades (vide Figura 2).
A Figura 3 exemplifica perfis de velocidade obtidos por ambas as técnicas ao longo do plano AA, a partir do qual se observa, principalmente, o desenvolvimento do jato oriundo da porta de maior diâmetro. Neste caso, observa-se que o mesmo se espalha após atingir o flange oposto, gerando recirculações próximo ao menisco e da saída do molde, semelhante ao fluxo descrito por Gabriel et al. [4]. $O$ jato da base tem uma profundidade de penetração de aproximadamente $200 \mathrm{~mm}$ a partir da qual se espalha em direção à alma e da superfície do flange no qual a SEN está posicionada. $O$ diâmetro da porta inferior avaliado neste caso, foi de $10 \mathrm{~mm}$.

A Figura 4 compara a intensidade de oscilação da superfície livre da válvula com 3 portas na saída e a válvula com quatro portas, sendo o diâmetro da porta da base igual a $10 \mathrm{~mm}$. Percebe-se, neste caso, uma ligeira redução na oscilação superficial, principalmente na região do flange oposto à válvula submersa.

Aumentando o diâmetro da porta da base de $10 \mathrm{~mm}$ para $16 \mathrm{~mm}$ observa-se uma maior representatividade deste nas características do fluxo, uma vez que a alimentação de fluido se torna mais homogênea ao longo do molde. A Figura 5 compara a dispersão de traçador obtida via CFD para ambos os diâmetros da porta inferior. Percebe-se que o aumento no diâmetro tende a equalizar a distribuição do fluido, reduzindo a assimetria do fluxo de fluido e consequentemente a de fluxo térmico. No sistema com

\section{4 portas - PIV}

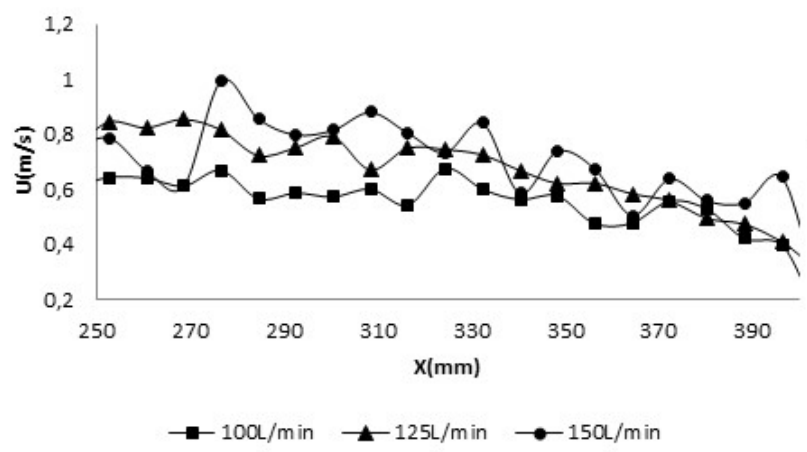

4 portas - CFD

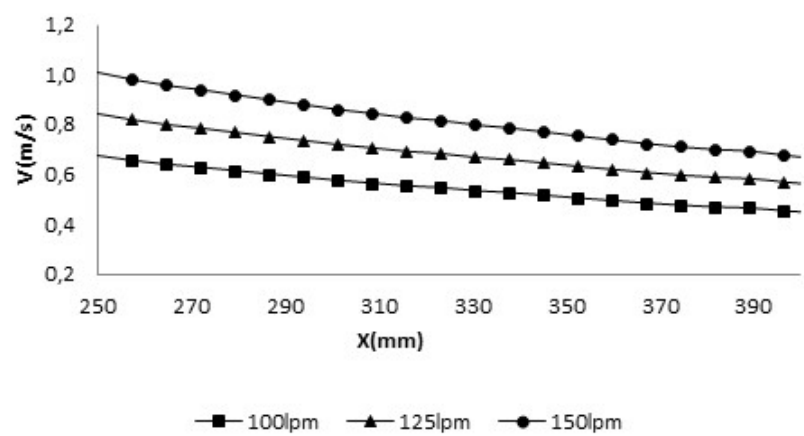

\section{3 portas - PIV}

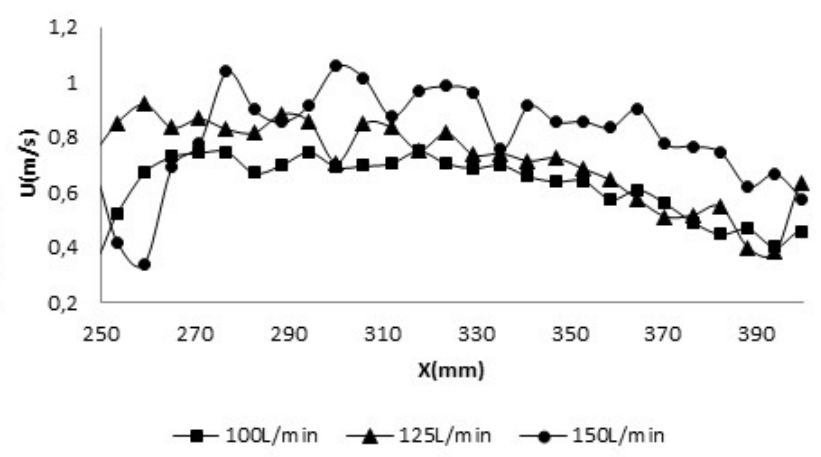

3 portas - CFD

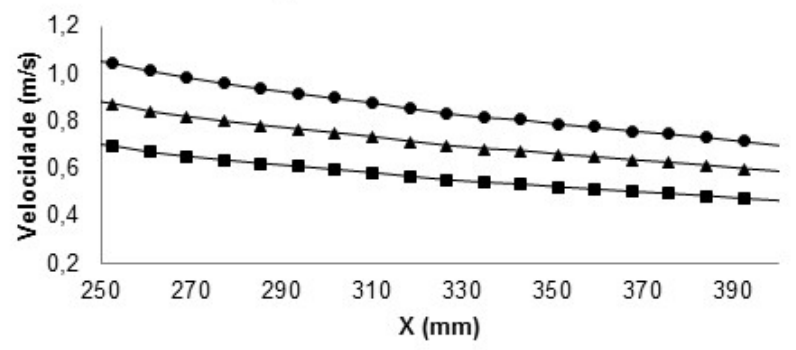

$\rightarrow-100 \mathrm{~L} / \mathrm{min}$ e imersão $=250 \mathrm{~mm} \longrightarrow-125 \mathrm{~L} / \mathrm{min}$ e imersão $=250 \mathrm{~mm}$

$\rightarrow 150 \mathrm{~L} / \mathrm{min}$ e imersão $=250 \mathrm{~mm}$

Figura 2. Comparação dos valores de velocidade ao longo da linha I, para válvula com 4 e 3 portas na saída respectivamente, obtidos pelas técnicas PIV e CFD. 


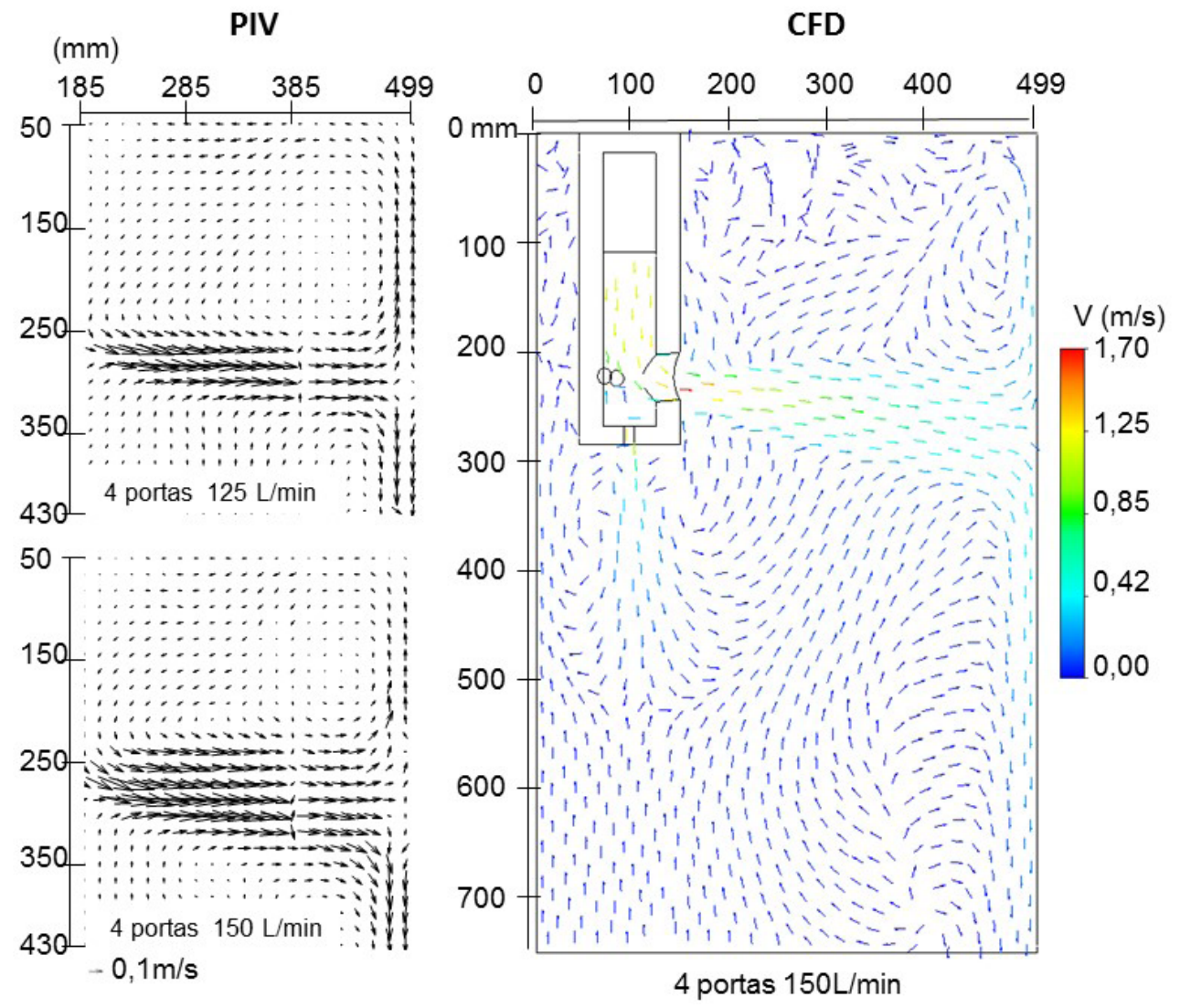

Figura 3. Perfis de velocidade obtidos pela técnica PIV e CFD.

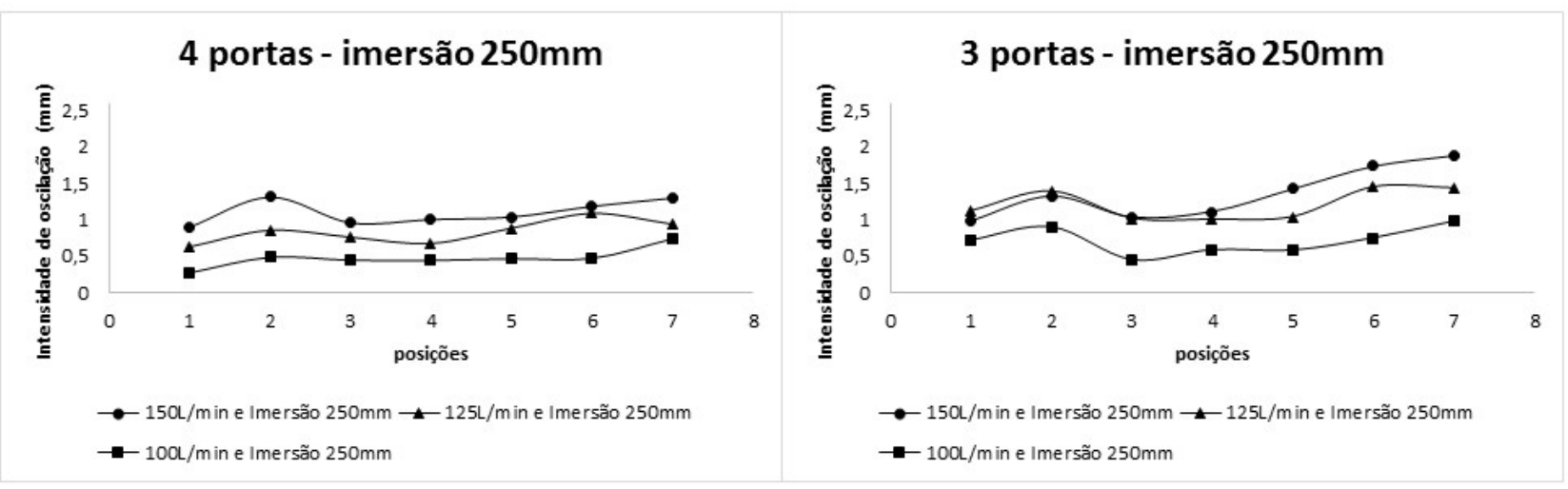

Figura 4. Comparativo da oscilação superficial obtido pelo sistema de alimentação composto por três e quatro portas de saída.

porta inferior de $16 \mathrm{~mm}$, percebe-se que ambos os flanges são preenchidos simultaneamente. Lee et al [9] mostraram que quando o aço a maiores temperaturas alimentados pela SEN, se distribui mais uniformemente ao longo do molde tem-se um desenvolvimento mais igualitário da casca solidificada. A formação de casca com espessura mais homogênea diminui a ocorrência de defeitos superficiais.

Os jatos oriundos das portas laterais de menor diâmetro incidem próximos à quina dos flanges, enquanto o jato da porta de maior diâmetro passa pela alma e impinge sobre o centro do flange oposto (Figura 6 a e b). A velocidade máxima do jato menor não foi significativamente influenciada pela inserção da porta na base da SEM (Figura 6a). Já no jato principal percebe-se uma redução na velocidade para uma mesma vazão de fluido, (Figura 6b). Estas regiões de máxima velocidade são propensas a ocorrência de problemas devido à má formação de pele, como rompimento de veio e trincas, conforme relataram Beaton et al. [I0] e De Santis. [I I]. 


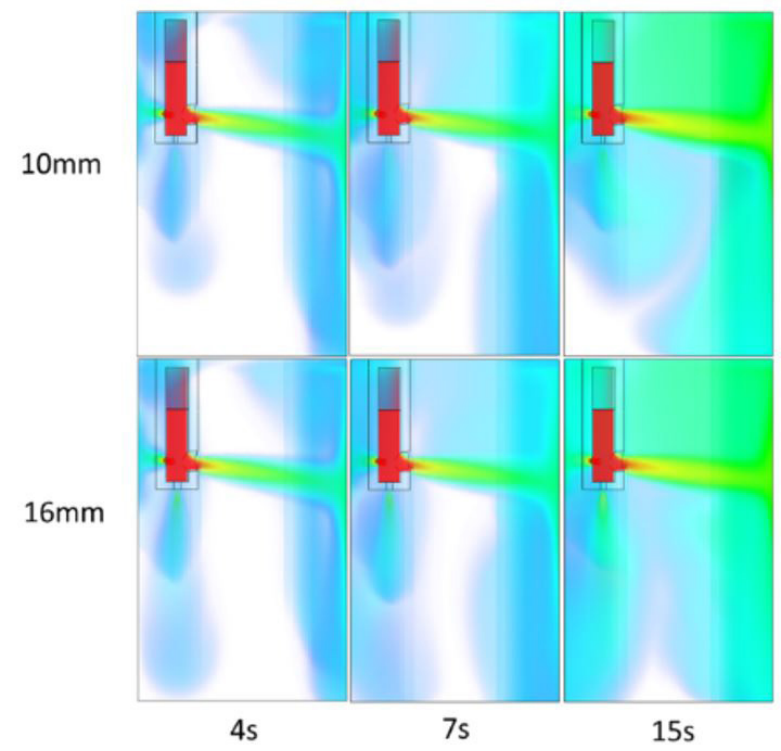

Figura 5. Análise da dispersão de traçador obtida via CFD, comparando o perfil de fluxo para diferentes diâmetros da porta inferior ( $10 \mathrm{~mm}$ e $16 \mathrm{~mm})$. Vazão de fluido $150 \mathrm{~L} / \mathrm{min}$.

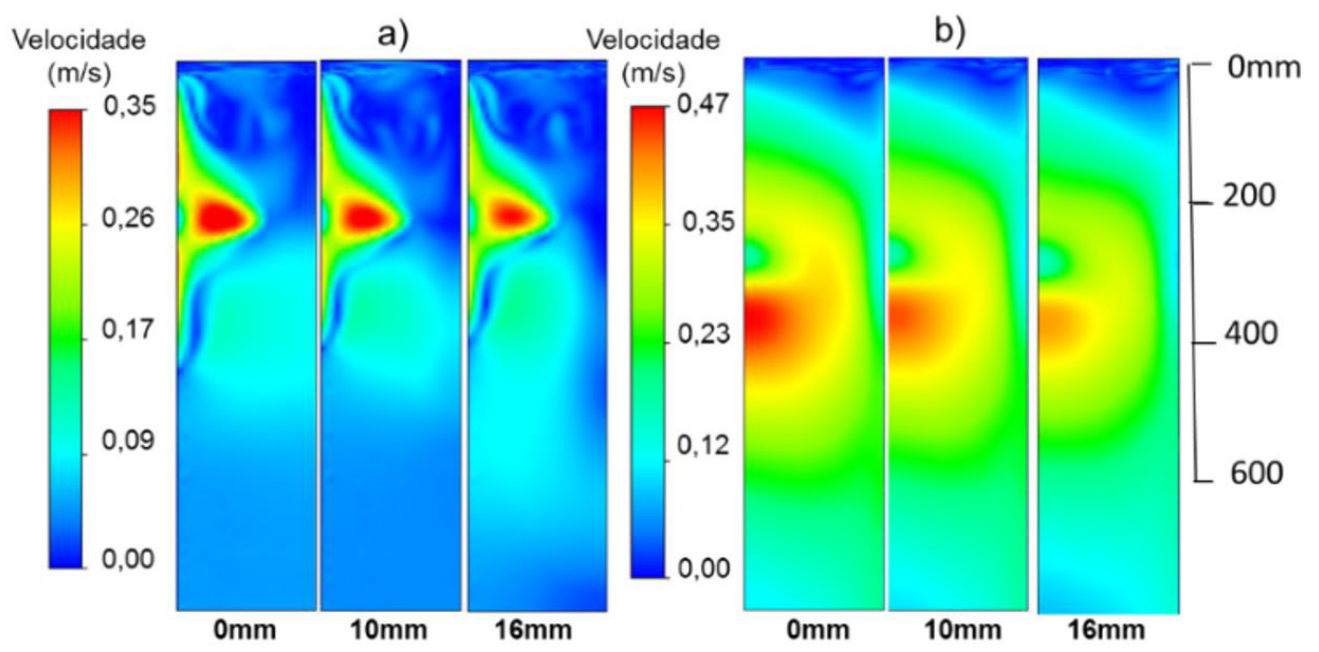

Figura 6. Valores de velocidade obtidos via CFD, no ponto de impingimento dos jatos em função do diâmetro da porta inferior. Vazão de fluido $150 \mathrm{~L} / \mathrm{min}$. a) jato da porta lateral com menor diâmetro - plano BB b) jato principal plano CC.

\section{CONCLUSÕES}

Os resultados obtidos forneceram as seguintes informações:

- O aumento na vazão de fluido promove aumento na intensidade de flutuação superficial.

- A válvula submersa com três portas laterais e um quarto furo na base com $10 \mathrm{~mm}$ de diâmetro, tem perfil de velocidade e distribuição de fluido semelhante à válvula com apenas 3 portas laterais.

- Aumentando o diâmetro da porta inferior para $16 \mathrm{~mm}$ observa-se maior alteração no perfil de velocidade, com distribuição mais homogênea de fluido.
- O aumento da porta inferior reduz a velocidade máxima de impacto do jato principal, reduzindo riscos de refusão.

- O diâmetro da porta inferior não interfere significativamente na velocidade do jato que sai das portas laterais menores.

\section{Agradecimentos}

Os autores gostariam de agradecer FAPEMIG, à Coordenação de Aperfeiçoamento de Pessoal de Nível Superior (CAPES) pelo apoio financeiro, em especial pelas bolsas de pesquisa concedidas a W.V. Gabriel, G.L. Alves e J. J. M. Peixoto, respectivamente. 


\section{REFERÊNCIAS}

I Peixoto JJM, Gabriel WV, Ribeiro LQ, Silva CA, Silva IA, Seshadri V. Computational and physical simulation of fluid flow inside a beam blank continuous casting mold. Journal of Materials Processing Technology. 2016;233:89-99.

2 Zhang L, Chen D, Long M, Xie X, Zhang X, Ma Y. Hydraulic simulation of fluid flow in beam blank continuous casting mold with double nozzles. In: Yurko J, Zhang L, Allanore A, Wang C, Spangenberger JS, Kirchain RE, Downey JP, May LD, eds. EPD Congress 2014. Hoboken: Wiley; 2014. p. 375-384.

3 Onishi M, Ueda T, Shinjo Y, Mizota H, Yao M, Fujimura T. Continuous casting of beam blanks. Kawasaki Steel Technical Report. 1981;3:13-25.

4 Gabriel WV, Peixoto JJM, Alves GL, Silva CA, Silva IA, Seshadri V. Fluxo de liquido num molde de beam blank e a remoção de inclusões. In: 48 Seminário de Aciaria, Fundição e Metalurgia de Não-Ferrosos; 2017 Outubro 2-6; São Paulo, Brasil. São Paulo: ABM; 2017. p. 299-309.

5 Yang J, Du Y, Shi R, Chui X, Liu C. Effect of SEN parameters on 3D flow field in mould of Beam Blank continuous casters. Iron and Steel Research International. 2004; I I (6): 19-23.

6 Chen W, Zhang YZ, Zhu LG, Zhang CJ, Chen Y, Wang B-X, et al. Three dimensional FEM study of fluid flow in mould for beam blank continuous casting: influence of nozzle structure and parameters on fluid flow. Ironmaking \& Steelmaking. 20I2;39(8):560-567.

$7 \mathrm{Xu}$ M, Zhu M. Transport phenomena in a Beam-Blank continuous casting mold with two types of submerged entry nozzle. ISIJ International. 20I4;55(4):79I-798.

8 Gabriel WV, Peixoto JJM, Alves GL, Silva CA, Silva IA, Seshadri V. Comportamento da interface metal/escória em molde de Beam Blank. In: 48 Seminário de Aciaria, Fundição e Metalurgia de Não-Ferrosos; 2017 Outubro 2-6; São Paulo, Brasil. São Paulo: ABM; 2017. p. 456-467.

9 Lee J-E, Yoon J-K, Han HN. 3-dimensional mathematical model for the analysis of continuous beam blank casting using body fitted coordinate system. ISIJ International. 1998;38(2): I32-I4I.

10 Beaton JW, Sgro A, Burini A, Razza P, Azizola A. Beam Blank in submerged pour casting: danieli technology and experience. In: METEC \& 2nd Estad; 2015 June 15-19; Dusseldorf, Germany. Welfengarten: TIB; 20I5. p. I-6.

II De Santis M, Cristallini A, Rinaldi M, Sgro A. Modelling-based innovative feeding strategy for Beam Blanks mould casting aimed at as-cast surface quality improvement. ISIJ International. 20I4;54(3):496-503.

Recebido em: 20 Nov. 2017

Aceito em: II Jun. 2018 